\title{
A new and spontaneous animal model for ankylosing spondylitis is found in cynomolgus monkeys
}

Huanhuan JIA

Guangdong Laboratory Monitoring Institute

Meili CHEN

Guangdong Pharmaceutical University

Xiaoling LUO

Guangdong Pharmaceutical University

Gang HOU

Third Affiliated Hospital of Sun Yat-Sen University

Ting LUO

Guangdong Laboratory Animals Monitoring Institute

Jin'e LIAO

Guangzhou Blooming-Spring Biological Research Institute

\section{Yongfeng LI}

Guangzhou Blooming-Spring Biological Research Institute

\section{Wei CHEN}

Guangdong Pharmaceutical University

\section{Jun CHEN}

Guangdong Pharmaceutical University

\section{Qingnan LI}

Guangdong Laboratory Animals Monitoring Institute

\section{Kai Kei MIU}

Chinese University of Hong Kong

\section{Ren Huang}

Guangdong Laboratory Animals Monitoring Institute

\section{Li LU (D1510558736@qq.com )}

Guangdong Pharmaceutical University https://orcid.org/0000-0002-7639-1891

\section{Research article}

Keywords: Ankylosing spondylitis, spontaneous animal model, cynomolgus monkeys, Non human primate 
Posted Date: May 17th, 2020

DOI: https://doi.org/10.21203/rs.3.rs-25995/v1

License: (9) This work is licensed under a Creative Commons Attribution 4.0 International License. Read Full License

Version of Record: A version of this preprint was published at Arthritis Research \& Therapy on January 3rd, 2022. See the published version at https://doi.org/10.1186/s13075-021-02679-5. 


\section{Abstract \\ Background}

Ankylosing spondylitis (AS) is a progressive, disabling joint disease that affects millions worldwide. There is a lack of useful disease model to conduct comprehensive mechanistic studies. Non-human primate model is a potential spontaneous model for ankylosing spondylitis (AS).

\section{Methods}

A group of cynomolgus monkeys with abnormal joints and abnormal movements were screened out from about 20,000 cynomolgus monkeys primarily. A 2-years follow-up study of this group of cynomolgus monkeys having joint lesions reported of spinal stiffness was performed by conducting hematological testing, radiographic examination, family aggregation analysis, pathological analysis and genetic testing.

\section{Results}

These diseased animals suffered from spontaneous AS with clinical features recapitulating human AS disease progression. The spontaneous incidence rate and the onset age of AS in monkeys similar to epidemiological features of AS patients. The disease progression in disease monkeys have shown bone erosion, osteophyte formation and "Bamboo-like" change. ESR, CRP IL-17, TNF-a and VEGF levels significantly increase in AS monkeys comparing with normal monkeys. Several features similar to AS patients, including cartilage destruction, cartilage ossification, chondroid metaplasia and bone formation were shown in AS monkeys pathological examination results. Both the serum bone resorption and bone formation biomarkers of AS monkeys were significantly reduce. In our current SNP sequencing results, loci that similar to AS-relative SNPs in human genome have not yet been found.

\section{Conclusions}

The study offers a promising non-human primate model for spontaneous ankylosing spondylitis which may serve as an excellent substitute for its pre-clinical research.

\section{Background}

Ankylosing spondylitis (AS) is a chronic progressive and disabling disease that affects the axial and peripheral joints. Clinically, a patient with AS would be described as suffering an inflammatory type of spinal pain which surmounted to immobility, followed by structural and functional impairments with sharp decrease in quality of life. AS is a disease that usually affects young people, where male are more often affected than female, with an odds ratio of roughly 2:1. Patients are mostly $15 \sim 30$ years old; while children and those over 40 years old are rare [1]. The incidence rate of AS in Chinese population is about 
$0.24 \%$ [2]. After years of active clinical research on AS, there is currently a clearer picture on its pathological features. The AS pathology were predominantly related to the entheses along three distinctive features in situ, i.e. inflammation, bone erosion and syndesmophyte (spur) formation[3]. However, the etiology of AS remains unclear, aside from suggested evidence in both genetic and environmental risk factors [4-6]. Failure to clarify the etiology of AS accounted for the bottleneck of drug development. Indeed, there is no drug available as a cure for AS at present. Clinically prescribed drugs for palliative treatment included non-steroidal anti-inflammatory drugs (NSAIDs), conventional antirheumatic drugs and biological agents, etc. These drugs were adopted for their potency in reducing inflammation and associated pain, however shall not prevent further ligament calcification and syndesmophyte formation along AS development. Obviously, their long-term usage shall trigger undesirable adverse reactions.

At present, most of the key findings for AS stemmed from clinical research. In clinical AS patients, diagnosis and staging relied on longitudinal imaging analysis (MRI and X-ray) to monitor disease progression, but the resolution and sensitivity of these imaging methods are inadequate. Collecting biopsy samples from spinal lesions in AS patients asssisted in elucidating the pathological mechanisms which included bone erosion and syndesmophyte formation [7-9]. These studies were incomplete as a whole since they were limited by sample size and matching anatomical location, and are primarily obtained from patients with advanced disease who have already undergone osteoproliferative transitions. Moreover, there are technical challenges in elucidating the mechanism of axial AS skeletal progression. It is therefore necessary to look for experimentally-feasible alternative subjects which spontaneously develop clinical features resembling that of AS patients.

Without its clear etiology, it is difficult to benchmark and produce disease animal model with high similarity to clinical AS patients. The lack of standardized models in turn hindered research in the pathogenesis of AS for therapeutics design. The currently adopted AS models are mainly based on rodents, including HLA-B27 humanized transgenic mouse model[10], ERAP1 deficient mouse model[11], or inflammation-inducing mouse models[12]. The HLA-B27 transgenic mice recapitulated the genetic susceptibility aspect of HLA-B27 in the pathogenesis of AS, but the model itself is intrinsically void with an inconsistent pathology. More importantly, in addition to joint lesions, the HLA-B27 transgene shall evoke lesions in other organs where symptoms are not accounted for as in AS patients. With the widen scope of research in the underlying mechanism of AS, researchers began to pay attention to the role of either infection or auto-inflammation in AS. Attempts in creating animal models of inflammation for disease induction received marginal success with almost certainly undesirable inflammation elsewhere [12]. These non-AS manifestations hampered the evaluation of drug effectiveness in treating AS. In summary, the existing experimental subjects do not reflect features in human AS nor its pathogenesis with additional drawbacks in specificity and reproducibility.

Significant efforts were made in screening more than 20,000 cynomolgus monkeys within the Guangdong Province such that we found cynomolgus monkeys which spontaneously developed spinal deformity and immobility between the ages of $4-12$ years old. Our preliminary $x$-ray imaging allowed 
pilot examination into the lesions of those cynomolgus monkeys carrying features similar to that of AS patients. We further conducted a two-year follow-up examination of these cynomolgus monkeys with a focus in spinal deformity, backed by cross-comparative examination in hematological indicators, imaging (X-ray, MRI and CT), family recurrence analysis, genetic testing and pathological analysis. The results showed that the characteristics, symptoms and pathogenesis of cynomolgus monkeys with these spinal deformities were similar to those patients with AS diagnosis with strikingly consistent pathological changes. Our report suggests cynomolgus monkeys with spinal deformity to be used as a spontaneous non-human primate pre-clinical model of AS.

\section{Materials And Methods}

\section{Animals}

All animals for the initial screen are adopted from Guangdong Chunsheng Biotechnology Development Co., Ltd., license number: SCXK (Guangdong) 2014-0027. The animals were housed under room temperature not higher than $30^{\circ} \mathrm{C}$ with a relative humidity fluctuating around $60 \%$, operating under at $12 \mathrm{~h} / 12 \mathrm{~h}$ normal light/dark cycle illumination. All procedures accorded with the guidelines of the Animal Care and Use Committee of the Guangdong Laboratory Animals Monitoring Institue (the reference number: GDLAMI-IACUC2018006).

A universal goniometer was used to evaluate the curvature of spine and the range of motion (ROM) of knee joint and hip joint to assist in screening for spontaneous AS monkeys in the population under the standard procedures described in details elsewhere [13].

\section{X-ray examination and CT scan}

Anteroposterior and lateral X-ray examinations of the spine and peripheral joints of all animals were documented in the initial screening. Subsequent CT scans of the whole pelvis shall be performed to animals requiring assessment of the hip joints. X-ray examination at the cervical, lumbar and caudal spine were performed using Mikasa HF400VA anatomical programming diagnostic X-ray system(Mikasa, Japan) when spinal assessment was required. CT and plain radiographs of the entire skeletal framework were obtained on the same day in both AS groups and control groups. $0.5 \mathrm{~mm}$-thick CT section images were obtained on an Aquilion 64 scanner (Toshiba, Japan) with tube current and potential set at $250 \mathrm{~mA}$ and $135 \mathrm{kV}$ respectively. X-ray images taken were compared among different time points to monitor the progressive deterioration along the disease.

\section{Hematological examination}

Complete blood count included hemoglobin (HGB), red blood cell (RBC), white blood cell (WBC), mean corpuscular hemoglobin concentration (MCHC) and platelets (PLT) values obtained from measurement 
collected via an automatic blood counter (Sysmex, XN-1000V, Japen). Erythrocyte sedimentation rate (ESR) was measured by the Westergren method. Biochemical estimations included sodium, potassium, urea, alkaline phosphatase (ALP), bilirubin, albumin(ALB), total protein (TP), globulin (GLOB), calcium $(\mathrm{Ca})$ and phosphorus $(\mathrm{P})$ were obtained from measurements performed by an automatic biochemical analyzer (HITACHI, 3100, Japen).

\section{Cytokines Detection}

Cytokine serum levels were measured in AS and control groups as follows: blood samples were taken at the time of clinical assessment and samples of sera were kept frozen at $-80{ }^{\circ} \mathrm{C}$ until performing the assay. IL-2, IL-6, IL-10, IL-12, IL-17, IFN- $y$, MCP-1, Tumor necrosis factor a (TNF-a), Vascular endothelial growth factor (VEGF), C-reactive protein (CRP), anti-cyclic peptide containing citrulline antibody (anti-CCP antibody), anti-Streptolysin O (ASO), Procalcitonin (PCT) and rheumatoid factors ( IgG-RF \& IgM-RF) levels were each measured with specific ELISA kits, in accordance with the manufacturer's protocol. In each assay, the appropriate recombinant human cytokine was used to generate the standard curve. Bonespecific alkaline phosphatase (BAP), parathyroid hormone (PTH), type I collagen amino-terminal propeptide (PINP), $\beta$-C-terminal telopeptides of type I collagen ( $\beta$-CTx), 25-Hydroxyvitamin (D325$(\mathrm{OH}) \mathrm{VD} 3)$, osteocalcin $(\mathrm{OC})$ and calcitonin were each measured with specific electro-chemiluminescence immunoassays.

\section{Paraffin Section And Staining}

Lumbar vertebra and caudal were decalcified with EDTA, followed by dehydration and embedding in paraffin blocks. Embedded lumbar vertebra and caudal samples were cut into $4-\mu \mathrm{m}$ serial sections along the sagittal plane using a standard rotary microtome. Sections were stained with Mayer's haematoxylin and eosin (H\&E) or safranin-0/fast green (Sigma-Aldrich, $0.1 \%$ safranin-0; $0.05 \%$ fast green in pure water) and then mounted. An Olympus BX51 microscope coupled to a computer with a imaging software, Bioquant OSTEO 2009(Bioquant Corporation1, USA), were used to capture overview images.

\section{SNP Genotyping}

Genomic DNA was extracted from peripheral blood using a Whole Blood Genomic DNA Extraction Kit (Takara) according to manufacturer's instructions. DNA samples were stored at $-20^{\circ} \mathrm{C}$ before genotyping. The genotyping of SNPs determined by the SNaPshot Assay(Shanghai Generay BioTech Co, Ltd) using the following primers: ERAP1 (sense, 5'- GGGCTGGATGTGAAAACCAT - 3'; antisense, 5'-

ATCACAGTGAGGGGAAGGA AT -3'; 84 bp, extension primer: TGATGAACACTTGGACACTGCAGA); IL23R (sense, 5'- ACGTGAGAATTCCA GGAGC - 3'; antisense, 5'- AAGCATGTTCCACCTTCAGA - 3'; 96 bp, extension primer: CTGACTGACTGATAAATTTTAGCCATTCTTCTGCCT); MHC-B (sense, 5'- 
primer: CTGACTGACTGACTGACTGACTTACAT GTCTTTGTACCTACT). The three SNPs of were genotyped using an ABI 3730XL Genetic Analyzer and the Multiplex SNaPshot system (Applied Biosystems, Foster City, CA). Genotypes of the SNPs were analyzed using the GeneMapper software (Applied Biosystems, Foster City, CA).

\section{Statistics}

All values are presented in the form of mean \pm SD. Data were analyzed with SPSS (Version 20.0) for Windows. The normality of distributions of variables was tested using the Kolmogorov-Smirnov test. Comparisons of normal distributed study parameters are performed using one-way ANOVA. P-values < 0.05 were considered statistically significant.

\section{Results}

\section{Physical examination}

The cynomolgus monkeys with spontaneous joint abnormalities generally develop symptoms such as hunchback, muscle atrophy, lameness, tail stiffness, restricted spinal activity and higher spinal rigidity. Both spine and the peripheral joints of the diseased monkeys were found to be swollen and immobile(Figure 1A). Most of the diseased monkeys have enlarged, stiff knees with limited mobility and are unable to stretch properly. All diseased monkeys' tails are stiff and have a bamboo-like exterior.

After monitoring closely in the animals' postures and movements, we further measured the mobility of their joints as depicted in the images (Fig. 1B). The curvatures of the spine of diseased monkeys are significantly higher than that of the normal. When compared to the control group, the curvatures of bilateral knee joints were significantly reduced, while the stretches were significantly increased; the curvatures of the right hip joint were significantly increased, the bilateral hip extensions were significantly reduced. There were no significant differences in the curvature and extension of bilateral elbow joints. It is shown that the lesion significantly changes the mobility of these joints.

\section{Incidence And Family Aggregation}

Through physical examination, we screened out nearly 100 animals with abnormal joints and abnormal movements from about 20,000 cynomolgus monkeys, further confirmed that those animals possessed spinal lesions though X-ray examination and biochemical testing. Refined identification allowed 57 cynomolgus monkeys to be diagnosed with AS, and the incidence rate was about $0.275 \%$ in the farm, which is similar to the likelihood as in human beings. Among these AS cynomolgus monkeys, 19 were males (33.33\%) and 38 were females (66.67\%) (Fig. 1C). Those AS monkeys were clustered between 4 and 10 years old (38 monkeys), which is effectively converted to human beings from 16 to 40 years old. There are 6 AS monkeys (10.53\%) which are less than 4 years old, which is considered the pre-adolescent 
period of cynomolgus. The youngest diseased animal we diagnosed was 2 years old, converted to about 8 years old as in human beings. There were 13 AS cynomolgus monkeys older than 10 years old $(22.81 \%)$ with generally more severe symptoms.

We further checked the animal archives to link up for the husbandry information and found that some AS monkeys were in fact close relatives (Fig. 1D). It was found that 5 AS cynomolgus monkeys $(5 / 57,8.77 \%)$ had family aggregation distributed in 2 separated bloodlines. The family tree of family 1 shown in Fig. 1 clearly indicated that two granddaughters of a single male cynomolgus monkey have ankylosing spondylitis. In family 2, two grandchildren of a female cynomolgus monkey were diagnosed with ankylosing spondylitis, while the uncle of this female AS cynomolgus monkey also developed ankylosing spondylitis.

\section{X-ray Examination And CT Scan}

Radiographs reveal erosive changes at the corners of the vertebral bodies in the early stages of disease together with outgrowth of bony spurs known as syndesmophytes in the later stages. When these syndesmophytes and their adjacent vertebral bodies are fused together, the spine shall appear as a single piece and is aptly described as bamboo spine. Similar lesions can also be found for knee joints, wrist joints and at the vertebral of the tails. CT scan had indicated a general rough surface for sacroiliac joints which are fused in severe cases ( Fig. 2A). X-ray examination showed that 57 cynomolgus monkeys had different degrees of joint abnormalities in the central joint, tail joint and limb joints ( Fig. 2B). To characterize the disease progression in AS monkeys, the lesions frequencies of each joint across 2 years disease time were deduced by X-ray examination. In the first physical and X-ray examination, 46 abnormal animals were found. The lesions frequencies of each joint at the first examination were summarized in Table 1. However, during the fourth examination, there was an obvious aggravation in disease symptoms. All 57 AS animals were found to have abnormal axial and peripheral joints, especially in the spine and sacroiliac joint. The order of joint lesions frequency is: sacroiliac joint $(57 / 57)>$ knee joint $(55 / 57)>$ lumbar vertebra(53/57) > elbow joint(8/57) > wrist and ankle joint(6/57). The increase in lesions is suggestive to a debilitating nature of AS at these diseased joints, with a higher tendency of developing lesions at joints which would be subjected to more mechanical stress, while small joints such as fingers and toes were rarely affected. This is an overt distinguishing feature different from rheumatoid arthritis (RA). 
Table 1

Statistics of lesion involvement sites in AS cynomolgus monkeys

\begin{tabular}{|lll|}
\hline Involvement site & Before AS( $\mathbf{n}=\mathbf{4 6})$ & After AS( $\mathbf{n}$ = 57) \\
\hline Axial joint & & \\
\hline Sacroiliac joint & $29(63.0)$ & $57(100.0)$ \\
\hline Caudal vertebrae & $26(56.5)$ & $48(84.2)$ \\
\hline Lumbar vertebra & $23(50.0)$ & $53(93.0)$ \\
\hline Thoracic vertebra & $18(39.1)$ & $36(63.2)$ \\
\hline Cervical vertebra & $13(28.3)$ & $24(42.1)$ \\
\hline Peripheral joint & & \\
\hline Knee joint & $41(89.1)$ & $55(96.5)$ \\
\hline Elbow joint & $3(6.5)$ & $8(14.0)$ \\
\hline Wrist joint & $6(13.0)$ & $6(10.5)$ \\
\hline Ankle joint & $1(2.2)$ & $6(10.5)$ \\
\hline Toe joint & 0 & $3(5.3)$ \\
\hline Finger joint & 0 & $3(5.3)$ \\
\hline Note.Data presented as $\mathrm{n}(\%)$. & \\
\hline
\end{tabular}

Comparison of the diseased progressing in AS cynomolgus monkeys by radiographic examination and the time required

We performed X-ray examination of disease animals once every six months. We found that disease progression in these animals is largely consistent with that of AS patients. Following a period of bone loss, new bone forms as spurs, which bridges the vertebrae to be fused into a single unit (Fig. 2C). By comparing the X-ray images of two-years follow-up examination, we further calculated how long it takes for these AS cynomolgus monkeys to change from bone erosion period to bone formation period (Table 2). We found that it takes about 7 months to change from bone erosion to osteophyte formation, about 10 months to change from osteophyte formation to bony bridge, while the transition from bony bridge to bamboo-like structures shall take around 12 months. It is suggested that the time from bone erosion to osteophyte formation is shorter, and it takes much longer for the osteophyte formation to develop into the final bamboo-like change. This also explains the small number of animal samples in the bone erosion period when we evaluate the bone metabolic indices of AS animals in the later study. In addition, we also counted the time required for the development of different parts of the spine in the AS monkeys. It was found that it takes around 13 months for the AS monkeys with abnormal peripheral 
joints to develop abnormalities in axial joints. Furthermore, it takes around 8 months to develop from having abnormal lumbar vertebras to then affect the thoracic vertebras. Thereafter, it takes around another 12 months for the affected thoracic vertebras to develop into diseased cervical vertebras.

Table 2

Statistics of disease progresses in AS cynomolgus monkeys

\begin{tabular}{|lll|}
\hline Disease progresses & $\begin{array}{l}\text { AS(n= } \\
\mathbf{5 7 )}\end{array}$ & $\begin{array}{l}\text { Length of time } \\
\text { (Month) }\end{array}$ \\
\hline $\begin{array}{l}\text { Uncertain(There were abnormalities in both mid-axis and peripheral } \\
\text { joints when first examination) }\end{array}$ & $\begin{array}{l}40(70.2) \\
\text { Peripheral joints to mid-axis joints }\end{array}$ & $/$ \\
\hline Lumbar vertebra to thoracic vertebra & $17(29.8)$ & $13.07 \pm 6.63$ \\
\hline Thoracic vertebra to Cervical vertebra & 9 & $8.44 \pm 1.33$ \\
\hline Thoracic vertebra to lumbar vertebra & 3 & $12.54 \pm 1.08$ \\
\hline Erosion to osteophyte in the Vertebra & 2 & $9.00 \pm 1.41$ \\
\hline Osteophyte to bone bridge in the Vertebra & $17(29.8)$ & $7.63 \pm 1.67$ \\
\hline Bone bridge to bamboo spine & $10(17.5)$ & $10.40 \pm 4.67$ \\
\hline Note.Data presented as $\mathrm{n}$ (\%) & $6(10.5)$ & $12.50 \pm 3.99$ \\
\hline
\end{tabular}

\section{Cytokines Of Inflammation}

To further distinguish AS from other similar diseases, we checked the expression of serum cytokines in diseased animals (Fig. 3A). When compared to normal monkeys, AS monkeys showed a significant increase in ESR and CRP levels, which represents two important indicators of inflammatory activity. However, there were no significant change in MCP-1, IgM-RF, Anti-CCP antibody, PCT and ASO levels. These results suggested that animals may be excluded from rheumatoid arthritis and infectious arthritis. More importantly, serum cytokine examination revealed that IL-17 and TNF-a levels were significantly elevated in AS monkeys when compared to normal monkeys ( Fig. 3B ), while IL-1 3 , IL-2, IL-8, IL-6, IL-10,

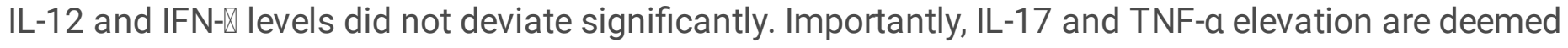
characteristic features of AS clinical patients. The above results suggest that the diseased animals demonstrated similar characteristics as in the AS patients in clinical settings.

\section{Hematological Examination}

Hematological examination showed that the PLT significantly increased, while HGB and MCHC significantly decreased in AS monkeys when compared to those in the control group, which indicated that the AS animal might have mild anemia symptoms ( Fig. 4 ). WBC increased significantly compared to the 
control group, indicating that the AS monkeys suffer inflammatory responses. Further cytology analysis in identifying individual leukocyte cell types revealed that neither leukocytes, eosinophils, basophils, nor monocytes were significantly expanded in population between AS group and control group, while lymphocytes were decreased and neutrophils were increased significantly in the AS group. It is suggested that the increase of WBC in AS cynomolgus monkeys was mainly due to the increase of neutrophils. Serum biochemical indicators showed that the levels of ALP, GLOB and TP in AS cynomolgus monkeys increased significantly when compared to the control group, while serum ALB, Ca and P decreased significantly. Changes in ALP, Ca, and $\mathrm{P}$ may be associated with changes in bone metabolismwhile changes in GLOB, TP, and ALB may be associated with nutritional malabsorption and chronic inflammation in AS animals.

\section{Pathological changes of lumbar and caudal vertebrae in AS animals}

The pathological changes of the caudal spine were illustrated in Fig. 5. The surface of the normal cynomolgus monkey caudal cartilage is intact and smooth, while inside the cartilage plate, chondrocytes are arranged tightly and orderly. However, the intervertebral space of the AS monkey caudal joints were much narrower when compared to the control ( Fig. 5B ); heterotopic ossification were observed in the cartilage. The cartilage was ossified from the intervertebral space side and inner layer. In severe cases, the cartilage was completely ossified. The remaining chondrocytes were irregularly arranged. Histological examination also demonstrated that the annulus fibrosus of normal disc were regularly arranged and filled with diffuse extracellular matrix, with spindlelike fibroblasts running parallel to these fibrils ( Fig. 5B ). By contrast, the annulus fibrosus of animals with AS were irregularly organized and did not contain any extracellular matrix. Furthermore, fewer fibrils were observed in these samples. Sporadically, the direct transformation of fibrous cells into woven bone was apparent ( Fig. 5B ).

In the AS cynomolgus lumbar vertebral sections, we found the following features, including cartilage destruction, chondroid metaplasia, bony spur formation, synovial hyperplasia, intra-articular fibrous stands, and vascular proliferation. These are all pathological features of AS patients observed on the clinical samples. The normal cynomolgus monkey intervertebral annulus fibrosus were arranged neatly, the chondrocytes in the cartilage plate were arranged in longitudinal rows and the cartilage plate was connected with the subchondral bone without erosion. The intervertebral disc space of the AS cynomolgus vertebral body gradually became narrow as the disease progressed, and the intervertebral space disappeared completely in the final stage when cartilage fusion.

In the early stage, thickening of the anterior ligament and synovial hyperplasia, can be observed, with inflammatory cell infiltration and obvious fibrous tissue hyperplasia( Fig. 5C). More importantly, there are a large amount of small blood vessels and osteoclasts in these fibrous tissues ( Fig. 5Ab and Fig. 5Ca ). The fibrous tissue was infiltrated by large numbers of monocytes ( Fig. 5Cc ). Small blood vessels also occurred in the bone marrow of AS monkeys, but rarely ( Fig. 5Cb ). We compared the vessels numbers of the bone marrow and ligaments of normal and monkey HE stained sections. The result is shown in Fig. 5C. The number of anterior ligament vessels of AS monkeys was significantly increased in 
comparison with normal monkeys, which indicated vascular invasion in the diseased disc. The destruction of the intervertebral disc cartilage plate can be observed at the early stage, with disc destruction combined with heterotopic bone formation. As a result, bony nodules can be observed in the deep-zone of the cartilage plate near the subchondral plate( Fig. 5A ).

The spur formation area is frequently situated around the anterior vertebral ligament during the syndesmophyte formation stage, where they extend from the vertebral body edge of the upper and lower sides of the disc, surrounding the proliferating fibrous tissue. Inside the spur formation area heterotopic ossification and chondroid metaplastic foci could be found ( Fig. 5Aa ). Cells inside the chondroid metaplastic foci, also known as calcified fibrocartilage, were not densely packed. Also, cells were rarely arranged in columns or hypertrophy like normal cartilage plates. The internal structure of the spur was similar to cancellous bone, and osteoblasts were attached to the bone surface. Finally, both the original and the ectopic cartilaginous tissues were replaced by bone at the cartilage fusion stage. Here, the original cartilages were fused, and chondral fusion was the predominant mode of ankyloses ( Fig. 5A).

\section{Bone Formation / Bone Resorption Markers}

We divided AS animals into two subgroups of bone formation group and bone erosion group according to their X-ray results. After biochemical analysis in determining the serum bio-marker levels of bone turnover, we found that three bone formation indicators (BAP, OC and PINP) and one bone resorption indicator ( $\beta$ CTx) were significantly reduced in AS animals when compared to the control ( Fig. 6 ). Moreover, there were no significantly changes of the above bone turnover markers between the bone erosion subgroup and bone formation subgroup. The 25-(OH)VD3 content was upregulated with trends similar to the bone turnover serum bio-markers. In contrast, PTH and calcitonin-two important hormones in bone metabolism-had no significant change not only between the AS group and control group, but also demonstrated no change between two subgroups.

\section{SNaPshot Sequencing}

We performed genetic analysis for the 20 AS-related SNPs that have been reported in literature [14-16]. The first step is to determine whether there existed nucleotide changes between the reference genome sequences of human and cynomolgus monkeys in these reported SNPs. It was found that among these reported AS-related SNPs, 10 SNPs (rs30187, rs11209026, rs1004819, rs11465804, rs10889677, rs1495965, rs6556416, rs2297909, rs11249215, rs11616188)are identical to the reference gene sequence of human and cynomolgus monkeys. We further confirmed whether these SNPs are located on the exons of the gene. As shown in the Table 3, only 4 SNPs are located in the exon region. However, the two HLArelated SNPs, rs4349859 and rs13202464, which have the highest OR, are not in the exon region of the HLA-B gene. Since HLA-B27 was recognized as important effector of AS, we chose rs4349859 (corresponding loci: Macaca_fascicularis_5.0:4:139402704) for SNP shot sequencing though rs4349859 is located on the introns. Among the 4 SNPs located in exons, rs30187 and rs10889677 are non- 
synonymous coding SNPs, we performed SNP shot sequencing on rs30187 and

rs10889677(corresponding loci: Macaca_fascicularis_5.0: chr6:95192910 and chr1:1600952276) in the genome of 55 monkeys (AS $=34$, control $=21$ ). 
Table 3

Consistency between Homo sapiens and Macaca fascicularis for sNPs within previously confirmed risk loci

\begin{tabular}{|c|c|c|c|c|c|c|c|c|}
\hline $\begin{array}{l}\text { Homo } \\
\text { sapiens SNP }\end{array}$ & Gene & Ref & OR & $P$ & $\begin{array}{l}\text { Exon } \\
\text { variant }\end{array}$ & $\begin{array}{l}\text { Consist- } \\
\text { ency }\end{array}$ & $\begin{array}{l}\text { Nonsyno- } \\
\text { nymous }\end{array}$ & Reference \\
\hline rs27434 & ERAP1 & $A$ & 1.19 & $\begin{array}{l}5.3 \times \\
10^{-12}\end{array}$ & $\sqrt{ }$ & $x$ & $\sqrt{ }$ & [15] \\
\hline rs30187 & ERAP1 & $\mathrm{T}$ & 1.35 & $\begin{array}{l}1.8 x \\
10^{-27}\end{array}$ & $\sqrt{ }$ & $\sqrt{ }$ & $\sqrt{ }$ & [15] \\
\hline rs11209026 & IL23R & G & 1.48 & $\begin{array}{l}2.3 \times \\
10^{-17}\end{array}$ & $\sqrt{ }$ & $\sqrt{ }$ & $\sqrt{ }$ & [15] \\
\hline rs1004819 & IL23R & G & 1.20 & $\begin{array}{l}1.1 \times \\
10^{-5}\end{array}$ & $x$ & $\sqrt{ }$ & $x$ & [13] \\
\hline rs10489629 & IL23R & $\mathrm{T}$ & 0.83 & 0.00011 & $x$ & $x$ & $x$ & [13] \\
\hline rs11465804 & IL23R & $\mathrm{T}$ & 0.68 & 0.0002 & $x$ & $\sqrt{ }$ & $x$ & [13] \\
\hline rs1343151 & IL23R & G & 0.80 & $\begin{array}{l}1.0 \times \\
10^{-5}\end{array}$ & $x$ & $x$ & $x$ & [13] \\
\hline rs10889677 & IL23R & C & 1.30 & $\begin{array}{l}1.3 x \\
10^{-6}\end{array}$ & $\sqrt{ }$ & $\sqrt{ }$ & $\sqrt{ }$ & [13] \\
\hline rs11209032 & IL23R & G & 1.30 & $\begin{array}{l}7.5 \times \\
10^{-9}\end{array}$ & $x$ & $x$ & $x$ & [13] \\
\hline rs1495965 & IL23R & C & 1.20 & $\begin{array}{l}3.1 \times \\
10^{-6}\end{array}$ & $x$ & $\sqrt{ }$ & $x$ & [13] \\
\hline rs4349859 & HLA-B & G & 40.80 & $\begin{array}{l}<10^{-} \\
200\end{array}$ & $x$ & $\sqrt{ }$ & $x$ & [14] \\
\hline rs13202464 & HLA-B & G & 27.60 & $\begin{array}{l}<10^{-} \\
200\end{array}$ & $x$ & $x$ & $x$ & [15] \\
\hline rs4389526 & ANTXR2 & $A$ & 1.15 & $\begin{array}{l}9.4 \times \\
10^{-8}\end{array}$ & $x$ & $x$ & $x$ & [15] \\
\hline rs6556416 & IL12B & C & 1.18 & $\begin{array}{l}1.9 \times \\
10^{-8}\end{array}$ & $x$ & $\sqrt{ }$ & $x$ & [15] \\
\hline rs2297909 & KIF21B & G & 1.14 & $\begin{array}{l}5.2 \times \\
10^{-12}\end{array}$ & $x$ & $\sqrt{ }$ & $x$ & [15] \\
\hline
\end{tabular}

Ref., reference allele; $\mathrm{OR}$, odds ratio calculated using the discovery sample unless otherwise stated; Consistency, Identity between Homo sapiens and Macaca fascicularis. 


\begin{tabular}{|c|c|c|c|c|c|c|c|c|}
\hline $\begin{array}{l}\text { Homo } \\
\text { sapiens SNP }\end{array}$ & Gene & Ref & OR & $\mathbf{P}$ & $\begin{array}{l}\text { Exon } \\
\text { variant }\end{array}$ & $\begin{array}{l}\text { Consist- } \\
\text { ency }\end{array}$ & $\begin{array}{l}\text { Nonsyno- } \\
\text { nymous }\end{array}$ & Reference \\
\hline rs11249215 & RUNX3 & $A$ & 1.19 & $\begin{array}{l}9.2 \times \\
10^{-11}\end{array}$ & $x$ & $\sqrt{ }$ & $x$ & [15] \\
\hline rs11616188 & LTBR & $A$ & 1.21 & $\begin{array}{l}4.1 \times \\
10^{-12}\end{array}$ & $x$ & $\sqrt{ }$ & $x$ & [15] \\
\hline rs8070463 & TBKBP1 & $\mathrm{C}$ & 1.13 & $\begin{array}{l}5.3 \times \\
10^{-8}\end{array}$ & $x$ & $x$ & $x$ & [15] \\
\hline rs10440635 & PTGER4 & $A$ & 1.10 & 0.017 & $x$ & $x$ & $x$ & [14] \\
\hline rs10781500 & CARD 9 & $\mathrm{~T}$ & 1.08 & 0.041 & $x$ & $x$ & $x$ & [14] \\
\hline
\end{tabular}

The minor allele in AS group was considered as a risk factor. According to the results of SNP shot sequencing, there was no minor alleles detected in SNP genotyping of these loci in AS or control animals(Table 4). The genotypes of these sites are consistent with the reference genome, and also consistent in between AS and control groups. These results suggested that these 3 AS-related SNPS found in clinical studies may not be involved in the pathogenesis of AS cynomolgus.

Table 4

Association results for SNPs within previously confirmed risk loci

\begin{tabular}{|lllll|}
\hline Gene & SNP & Ref. & $\begin{array}{l}\text { AS group } \\
(\mathbf{n = 3 4 )}\end{array}$ & $\begin{array}{l}\text { Control group } \\
(\mathbf{n = 2 1})\end{array}$ \\
\hline ERAP1 & rs30187 & A & $34(100 \%)$ & $21(100 \%)$ \\
\hline IL23R & rs10889677 & C & $34(100 \%)$ & $21(100 \%)$ \\
\hline HLA-B & rs4349859 & C & $34(100 \%)$ & $21(100 \%)$ \\
\hline
\end{tabular}

\section{Discussion}

Epidemiological studies have found that the incidence of AS patients in China is about $0.2 \%-0.54 \%[2]$. Although the incidence rates are not fully consistent across countries, they are mainly between $0.3 \%$ and $0.5 \%$ [1]. Among the 20,000 cynomolgus monkey populations in the farm, 57 were diagnosed as suffering from AS; the spontaneous incidence rate of AS in monkeys was in line with that of clinical cases at about $0.28 \%$. In addition, the conversion relationship between human and monkey age ( 1 year old cynomolgus monkey is about 4 years old in human being) provided a rough estimate for the onset age of AS animals similar to epidemiological results. Moreover, genetic analysis of diseased animals indicates that the diseased animals are subjected to inheritance running along families. The above results indicate that the 
statistical data of the diseased animals we obtained are consistent with AS epidemiology. However, our statistical analysis failed to re-establish the gender disparity in these monkeys, probably subject to the biased housing population with predominantly female within the farm. In reality, male animals reaching 3-5 years old would be sold off, rendered it impossible to track their disease phenotypes. Therefore, we believe the current odds ratio overestimated the incidence in female with confounding bias in terms of gender susceptibility.

Radiographic examination forms the basis for clinical diagnosis of AS. Sacroiliitic joint lesion is a hallmark of AS, especially prominent in earlier disease stages, followed by multiple manifestations such as spinal and peripheral joint lesions. [1] The lesions of the vertebral body are the most distinctive, with overt pathological changes consisting of bone erosion and syndesmophyte formation. CT scan showed that the sacroiliitic joint surface of the AS cynomolgus monkey had rough joint surface, narrow joint space which even eventually diminished in the final stage. X-ray examination was sufficient to demonstrate the lesions at the spine. The bone erosion manifests as a square vertebra where the vertebral corner was eroded, and then the syndesmophyte grows from the adjacent vertebral corners. Finally, the adjacent vertebral bodies fuse together, resulting in a typical "Bamboo-like" spine. The above processes in monkeys matches perfectly with that along the progression of AS patients, which instigated a 2-year follow-up in these diseased monkeys.

After multiple X-ray examination over 2 years, we found that the majority of bone deterioration occurred within half a year where vertebral joints would undergo transformations from bone erosion to syndesmophyte formation in the diseased monkeys, which is much more accelerated when compared to humans. Nonetheless, the change from initial syndesmophyte formation to the final bamboo-like change requires a relatively longer time. The gradual deterioration at a shorter window to compared human is conducive to better evaluation for drug efficacy and pharmacology.

Clinically, it still lack of gold standard for the diagnosis of AS. It is difficult to distinguish AS from other similar diseases like rheumatoid arthritis (RA) only by radiographic examination in the early stage $[6,17]$. With a wider scope of research on AS in recent years, specific detection markers were identified for AS, including CRP [18], RF[19], IL-17[20, 21], TNF-a[22] etc. A combined $x$-ray imaging and marker changes of CRP, RF, IL-17, TNF-a detection provides a more comprehensive diagnostic account for diseased animals, so as to allow us to confirm the existence of a spontaneous AS cynomolgus monkey model.

Hematological examination is usually not regarded as a key indicator of clinical diagnosis of AS. This may be due to changes in hematological parameters that are mainly related to changes in inflammation in the body, and are difficult to distinguish from other diseases. However, changes in the animals' hematological indicators still reflect consistent clinical performance of AS patients. Previous studies have found that patients with AS have unchanged or elevated white blood cell counts[18], elevated alkaline phosphatase[23], elevated platelets[18], and decreased hemoglobin[24] and serum albumin[25]. Alkaline phosphatase is the most commonly used indicator for assessing bone formation and bone turnover and is one of the biomarkers that reflect the activity of osteoblasts. In the hematological analysis of AS 
cynomolgus monkeys, it was found that the changes of WBC and other indicators were consistent with the clinical reports. In addition, the percentage of lymphocytes decreased significantly, and the percentage of neutrophils increased significantly, which is consistent with previous studies of AS patients[18], indicating that neutrophils may play a role in the AS disease response. The serum levels of TNF-a, IL-6, IL-4, and VEGF in AS cynomolgus monkeys are elevated, and the increase in VEGF can be supported by the results observed in our pathological study. Although it has been pointed out in clinical studies that there are symptoms of vasospasm in the ankle joint of patients with AS, there is no report that indicated a rise in serum VEGF in AS patients [26]. Nonetheless, one study had suggested an elevated level of VEGF RNA in patient biopsy specimens [27]. Whether VEGF can be used as a specific diagnostic indicator for AS and how it is involved in the development of AS remains to be further studied. Moreover, our study also reported a significant decrease in serum calcium and phosphorus levels, which may be due to their involvement in calcification of the ligaments in the lesions where vast amounts were deposited at lesion site, leading to a drop in serum calcium and phosphorus levels. Clinical studies have reported that serum OPG levels in AS patients are significantly lower than that in healthy subjects, and subgroup analysis in AS patients found that OPG levels in AS patients were significantly higher in the active phase (Syndesmophyte formation ) and inactive period group [28]. This suggests that ectopic bone formation or bone erosion in AS patients should be associated with dysfunction of bone-regulating factors such as OPG at the cellular level. In our result, both the serum bone resorption and bone formation biomarkers of AS disease cynomolgus monkeys were significantly lower than the normal control group, which is consistent to the clinical findings. This may be due to the fact that these factors regulating bone turnover mainly by local microenvironment remodeling by regulation in osteoblasts and osteoclasts activities, so they are mostly concentrated in the lesions sites, resulting in a drop in global context. To confirm this argument we may need to conduct further immunohistochemistry analysis and bone morphometry in the lesions sites. However, in the subgroup analysis between the bone erosion and the syndesmophyte formation subgroups, it was found that there was no significant difference in serum bone resorption index and bone formation index between two subgroups; this may be due to the small number of animals in the bone erosion group. However, the bone metabolism index should reflect the change of the cell regulation level in vivo much earlier than the radiographic changes, which is ideal to suggest the response rate in drug intervention.

Based on the radiographic examination, we further analyzed the pathological changes of AS cynomolgus monkeys. Heterotopic ossification of the intervertebral disc cartilage was found in anatomical specimens with no obvious abnormalities in radiographic examination, indicating that the pathological examination revealed in vivo subtle lesions more early and accurately. Several features, including synovitis, cartilage ossification, bone formation and chondroid metaplasia, as well as cartilage destruction are commonly found in the joints of AS patients [7]. In early lesions, synovial thickening, fibrous tissue hyperplasia, vascular proliferation and cartilage destruction were found in the anterior ligament of the animal vertebral body. In the late stage, the vertebral body was more common in the formation of spurs, and the chondroid metaplasia foci were visible in the spur area. There was no obvious inflammatory cell infiltration, while the site was laden with active osteoblasts and osteoclasts. These observations have completely 
reproduced the pathological changes as observed in clinical AS patient specimens $[8,29]$ and have further reinstated the diagnosis of spontaneous AS in these animals. More valuably, we gathered new evidence in establishing controversial issues raised in prior animal research through careful observation of the whole vertebral body section in these monkeys.

The first issue is about the presence of vascularized fibrous tissue in the diseased joint, an outgrowth also known as granulation tissue, with an uncertain origin [7]. They are suggested to originate from the synovium or bone marrow however lack of clear evidence to support such claim. After analyzing the biopsy specimen, Bleid concluded that the fibrous tissue was from the bone marrow; they first erode the subchondr plate and then into the cartilage surface [8]. This may be because the specimens of the biopsy are too small to observe the changes in the entire vertebral body. We found that these fibrous structures appear more frequently between the synovial membrane and the disc annulus fibrosus, and at the edges of them, aggregate a large number of osteoclasts in the fibrous tissue. These osteoclasts are considered necessary for the degradation of the cartilage matrix. So we think these vascularized fibrous tissues are more likely to be derived from the synovium. The phenomena we described here have not been encountered in mice with progressive ankylosis[30], in HLA-B27 transgenic rats with experimental inflammatory disease [31, 32], in HLA-B27 transgenic mice[10], or in animals with murine ankylosing enthesopathy[33]. It should be pointed out that in the latter two animal disease models,no lesions of the spine were even present.

The second issue is about whether inflammation is accompanied by progression of AS. As shown our results, inflammatory cell infiltration is observed only in the early stages and rarely seen in the later stages. Our observation is supported by clinical report, which described MRI studies in AS patients that manifested higher incidence of syndesmophytes in vertebral corners with resolved inflammation [34] and $68 \%$ of syndesmophytes developed from vertebral corners without active inflammation during the 2-year observation period [35]. Note that the rough estimation may be largely qualitative and subject to accuracy bias related to limits in detection of inflammation by the MRI; our results is nevertheless in line with the prediction that in the vertebral joints during the syndesmophytes formation stage of AS, inflammatory cells rarely appear in the spur formation area. It is also possible that inflammatory cells appear in areas other than the joint ligaments and cause pain in the patient, and indeed, this should be carefully studied later. Therefore, we believe that inflammation is not critical to the bony spurs formation in the late stage of AS, and clinical study had shown that anti-inflammatory treatment in AS clinical patients cannot inhibit the progression of syndesmophytes formation [36]. Therefore, the way forward to AS drug design should be to identify lead compounds that may halt or even reverse the bone formation of syndesmophytes. Drug-induced mice [37] reported in the past have obvious inflammatory cell infiltration and ectopic cartilage formation in the intervertebral disc area, but rare spur formation. The inflammationinduced AS model is more manifested in the inflammatory response of the spine or peripheral joints [12]. Hence, we confirm that spontaneous AS cynomolgus monkeys have higher similarities with clinical patients relative to the currently-adopted inflammation-induced rodent models, in terms of mimicking human AS pathological changes and disease progression. This support the migration of research in AS to our spontaneous animal model to study its etiology and facilitate therapeutics discovery. 
The earliest definition of AS as a hereditary disease was mainly due to the higher recurrence risk of AS than general non-hereditary disease, with a $63 \%$ recurrence risk in monozygotic twins[38] and a $8.2 \%$ recurrence risk in first-degree relatives[39]. Through genome-wide association studies, more and more genetic loci have been found to be associated with AS[2, 40,41]. More than 100 genetic loci have been associated with AS, but they only summarized up to $30 \%$ of AS heritability.[5] Among those genes, HLAB27, ERAP1 and IL-23R received much attention, accounting for the population-attributable risks at approximately $90 \%, 26 \%$ and $1 \%$ respectively $[18,25]$. Interestingly, how these genotypes shall affect the pathogenesis of AS remains unknown.

In this study, 20 AS-relative SNPs were screened, and SNaPshot was performed on 3 SNPs. No AS risk allele SNPs were found in cynomolgus monkeys, indicating that the dangerous allele SNPs in AS is not consistent between cynomolgus monkeys and human beings, although these two species have high degree of similarity in genetic makeup. Our current result may be limited by the small pool and a restricted source in available animal samples. This result suggests that spontaneous AS cynomolgus monkeys reported in this study are not suitable for use in gene editing therapy research. However, previous studies have shown that AS is determined by genetic factors and environmental factors, and is affected by multiple genes. Therefore, methods for improving or treating AS through gene therapy should be quite difficult to implement.

In summary, there is a need to secure an animal model of AS disease to look into the pathogenesis of AS to push for the development of therapeutics. The rodent models obtained by inflammation induction or genetic editing are disappointing in recapitulating the pathogenesis of AS. The lack of guidance from actual etiology studies and heavy reliance in individual genetic factors or inflammatory effects also hindered the research in AS, which adds up to no yield in encouraging breakthrough results. Although the pathological studies obtained from clinical specimens may to some extent reflect the actual course of action along AS development, the source of samples remained scared and usually related to late stage of the disease. Furthermore, sample collection is almost always limited by ethical concerns and it remains hard to obtain a comprehensive account as much as information that could be gathered from our animal models.

\section{Conclusions}

In this study, we evaluated AS cynomolgus monkeys from aspects such as epidemiology, hematology, radiographic imaging, pathology, and genetic linkage analysis. We confirmed that our spontaneous AS model in cynomolgus monkeys demonstrated strikingly similar features to that in clinical AS patients, therefore, it represents an excellent novel animal model to pave the groundbreaking discovery of AS etiology and evaluating drug efficacy.

\section{Abbreviations}


AS: Ankylosing spondylitis; NSAIDs: non-steroidal anti-inflammatory drugs; ROM: the range of motion; WBC: white blood cell; HGB: hemoglobin; RBC: red blood cell; MCHC: mean corpuscular hemoglobin concentration; PLT: platelets; ALP: alkaline phosphatase; ALB: bilirubin, albumin; TP: otal protein; GLOB: globulin; Ca: calcium; P: phosphorus; TNF-a: Tumor necrosis factor a; VEGF: Vascular endothelial growth factor; CRP: C-reactive protein; anti-CCP antibody: anti-cyclic peptide containing citrulline antibody; ASO: anti-Streptolysin 0; PCT:Procalcitonin; BAP: Bone-specific alkaline phosphatase; PTH: parathyroid hormone; PINP: type I collagen amino-terminal propeptide; $\beta$-CTx: $\beta$-C-terminal telopeptides of type I collagen; D325-(OH)VD3: 25-Hydroxyvitamin; OC: osteocalcin; H\&E: haematoxylin and eosin; RA: rheumatoid arthritis; SNP: single nucleotide polymorphism

\section{Declarations}

\section{Ethics approval and consent to participate}

All primary studies indicated that ethics approval. All procedures accorded with the guidelines of the Animal Care and Use Committee of the Guangdong Laboratory Animals Monitoring Institue (the reference number: GDLAMI-IACUC2018006)

\section{Consent for publication}

Not applicable

\section{Availability of data and materials}

The datasets used and/or analysed during the current study are available from the corresponding author on reasonable request.

\section{Competing interests}

The authors declare that they have no competing interests.

\section{Funding}

The National Natural Science Foundation of China [Grant no. 81941010] and Science and Technology Planning Project of Guangdong Province of China [Grant no.2017A070701003, 2017B020231001 and 2019A030317009].

\section{Authors' contributions}

JIA Huanhuan, CHEN Meili , LUO Xiaoling, LU Li and HOU Gang designed the study, analyzed the data, and drafted the manuscript. LUO Ting, LIAO Jin'e, LI Yongfeng, CHEN Wei, CHEN Jun, LI Qingnan, MIU Kai Kei and Huang Ren participated in the data collection, and helped in the drafting of the manuscript. All authors contributed to discussions and read and approved the final manuscript. 
Acknowledgements

Not applicable

\section{References}

1. Braun J, Sieper J. Ankylosing spondylitis. Lancet. 2007;369:1379-90. doi:10.1016/S01406736(07)60635-7.

2. Lu X, Wang L, Chen S, He L, Yang X, Shi Y, et al. Genome-wide association study in Han Chinese identifies four new susceptibility loci for coronary artery disease. Nat Genet. 2012;44:890-4. doi:10.1038/ng.2337.

3. Ramiro S, van Tubergen A, van der Heijde D, Stolwijk C, Bookelman G, Dougados M, et al. Brief report: erosions and sclerosis on radiographs precede the subsequent development of syndesmophytes at the same site: a twelve-year prospective followup of patients with ankylosing spondylitis. Arthritis Rheumatol. 2014;66:2773-9. doi:10.1002/art.38775.

4. Tam LS, Gu J, Yu D. Pathogenesis of ankylosing spondylitis. Nat Rev Rheumatol. 2010;6:399-405.

5. Ranganathan V, Gracey E, Brown MA, Inman RD, Haroon N. Pathogenesis of ankylosing spondylitis recent advances and future directions. Nat Rev Rheumatol. 2017;13:359-67. doi:10.1038/nrrheum.2017.56.

6. Watad A, Bridgewood C, Russell T, Marzo-Ortega H, Cuthbert R, McGonagle D. The Early Phases of Ankylosing Spondylitis: Emerging Insights From Clinical and Basic Science. Front Immunol. 2018;9:2668. doi:10.3389/fimmu.2018.02668.

7. Francois RJ, Gardner DL, Degrave EJ, Bywaters EG.Histopathologic evidence that sacroiliitis in ankylosing spondylitis is not merely enthesitis. Arthritis and rheumatism. 2000,43:20112024.doi:10.1002/1529-0131(200009)43:9<2011::AID-ANR12>3.0.CO;2-Y.

8. Bleil J, Maier R, Hempfing A, Schlichting U, Appel H, Sieper J, et al. Histomorphologic and histomorphometric characteristics of zygapophyseal joint remodeling in ankylosing spondylitis. Arthritis Rheumatol. 2014;66:1745-54. doi:10.1002/art.38404.

9. Benjamin M, Toumi H, Suzuki D, Hayashi K, McGonagle D. Evidence for a distinctive pattern of bone formation in enthesophytes. Ann Rheum Dis. 2009;68:1003-10. doi:10.1136/ard.2008.091074.

10. Khare SD, Luthra HS, David CS. Spontaneous inflammatory arthritis in HLA-B27 transgenic mice lacking beta 2-microglobulin: a model of human spondyloarthropathies. J Exp Med. 1995;182:11538. doi:10.1084/jem.182.4.1153.

11. Pepelyayeva Y, Rastall DPW, Aldhamen YA, O'Connell P, Raehtz S, Alyaqoub FS, et al. ERAP1 deficient mice have reduced Type 1 regulatory $T$ cells and develop skeletal and intestinal features of Ankylosing Spondylitis. Scientific reports. 2018;8:12464. doi:10.1038/s41598-018-30159-5.

12. Bardos T, Szabo Z, Czipri M, Vermes C, Tunyogi-Csapo M, Urban RM, et al. A longitudinal study on an autoimmune murine model of ankylosing spondylitis. Ann Rheum Dis. 2005;64:981-7. doi:10.1136/ard.2004.029710. 
13. Norkin C, White D. Measurement of Joint Motion: A Guide to Goniometry, 4th ed. Philadelphia, PA: F. A. Davis Company. 2009; 241-262.

14. Evans DM, Spencer CC, Pointon JJ, Su Z, Harvey D, Kochan G, et al. Interaction between ERAP1 and HLA-B27 in ankylosing spondylitis implicates peptide handling in the mechanism for HLA-B27 in disease susceptibility. Nat Genet. 2011;43:761-7. doi:10.1038/ng.873.

15. Lin Z, Bei JX, Shen M, Li Q, Liao Z, Zhang Y, et al. A genome-wide association study in Han Chinese identifies new susceptibility loci for ankylosing spondylitis. Nat Genet. 2011;44:73-7. doi:10.1038/ng.1005.

16. Control WTrustC, Australo-Anglo-American Spondylitis C, Burton C, Clayton PR, Cardon DG, Craddock LR. N, et al. Association scan of 14,500 nonsynonymous SNPs in four diseases identifies autoimmunity variants. Nat Genet. 2007;39:1329-37. doi:10.1038/ng.2007.17.

17. Tobon GJ, Youinou P, Saraux A. The environment, geo-epidemiology, and autoimmune disease: Rheumatoid arthritis. Autoimmun Rev. 2010;9:A288-92. doi:10.1016/j.autrev.2009.11.019.

18. Bozan N, Alpayci M, Aslan M, Cankaya H, Kiroglu AF, Turan M, et al. Mean platelet volume, red cell distribution width, platelet-to-lymphocyte and neutrophil-to-lymphocyte ratios in patients with ankylosing spondylitis and their relationships with high-frequency hearing thresholds. European archives of oto-rhino-laryngology: official journal of the European Federation of Oto-RhinoLaryngological Societies (EUFOS) : affiliated with the German Society for Oto-Rhino-Laryngology Head Neck Surgery. 2016;273:3663-72.

19. Cowling P, Ebringer R, Ebringer A. Association of inflammation with raised serum IgA in ankylosing spondylitis. Ann Rheum Dis. 1980;39:545-9. doi:10.1136/ard.39.6.545.

20. Zambrano-Zaragoza JF, Agraz-Cibrian JM, Gonzalez-Reyes C, Duran-Avelar Mde J, Vibanco-Perez N. Ankylosing spondylitis: from cells to genes. International journal of inflammation. 2013; 501653.doi:10.1155/2013/501653.

21. Taams LS, Steel KJA, Srenathan U, Burns LA, Kirkham BW. IL-17 in the immunopathogenesis of spondyloarthritis. Nat Rev Rheumatol. 2018;14:453-66. doi:10.1038/s41584-018-0044-2.

22. Gratacos J, Collado A, Filella X, Sanmarti R, Canete J, Llena J, et al. Serum cytokines (IL-6, TNF-alpha, IL-1 beta and IFN-gamma) in ankylosing spondylitis: a close correlation between serum IL-6 and disease activity and severity. Br J Rheumatol. 1994;33:927-31. doi:10.1093/rheumatology/33.10.927.

23. Kendall MJ, Lawrence DS, Shuttleworth GR, Whitfield AG. Haematology and biochemistry of ankylosing spondylitis. British medical journal. 1973;2:235-7. doi:10.1136/bmj.2.5860.235.

24. Lin F, Wang X, Liang Y, Liu D, Zhang Y, Zhong R, et al. Red Blood Cell Distribution Width in Rheumatoid Arthritis, Ankylosing Spondylitis and Osteoarthritis: True Inflammatory Index or Effect of Anemia? Annals of clinical and laboratory science. 2018; 48: 301-307.

25. Hart FD, Maclagan NF. Ankylosing Spondylitis A. Review of 184 Cases. Ann Rheum Dis. 1955;14:77.doi. 10.1136/ard.14.1.77. 
26. Poddubnyy D, Conrad K, Haibel H, Syrbe U, Appel H, Braun J, et al. Elevated serum level of the vascular endothelial growth factor predicts radiographic spinal progression in patients with axial spondyloarthritis. Ann Rheum Dis. 2014;73:2137-43. doi:10.1136/annrheumdis-2013-203824.

27. Zhang Y, Hu X, Zhang C, Zhou Y, Chu TW. Suppression subtractive hybridization reveals differentially expressed genes in supraspinous ligaments of patients with ankylosing spondylitis. Mol Med Rep. 2015;11:4482-8. doi:10.3892/mmr.2015.3272.

28. Taylan A, Sari I, Akinci B, Bilge S, Kozaci D, Akar S, et al. Biomarkers and cytokines of bone turnover: extensive evaluation in a cohort of patients with ankylosing spondylitis. BMC Musculoskelet Disord. 2012;13:191. doi:10.1186/1471-2474-13-191.

29. Bleil J, Sieper J, Maier R, Schlichting U, Hempfing A, Syrbe U, et al. Cartilage in facet joints of patients with ankylosing spondylitis (AS) shows signs of cartilage degeneration rather than chondrocyte hypertrophy: implications for joint remodeling in AS. Arthritis research therapy. 2015;17:170. doi:10.1186/s13075-015-0675-5.

30. Mahowald ML, Krug H, Halverson P. Progressive ankylosis (ank/ank) in mice: an animal model of spondyloarthropathy. II. Light and electron microscopic findings. J Rhuematol. 1989;16:60-6.

31. Hammer RE, Maika SD, Richardson JA, Tang JP, Taurog JD. Spontaneous inflammatory disease in transgenic rats expressing HLA-B27 and human beta 2m: an animal model of HLA-B27-associated human disorders. Cell. 1990;63:1099-112. doi:10.1016/0092-8674(90)90512-d.

32. Rysnik O, McHugh K, van Duivenvoorde L, van Tok M, Guggino G, Taurog J, et al. Non-conventional forms of HLA-B27 are expressed in spondyloarthritis joints and gut tissue. J Autoimmun. 2016;70:12-21. doi:10.1016/j.jaut.2016.03.009.

33. Eulderink F, Ivanyi P, Weinreich S. Histopathology of murine ankylosing enthesopathy. Pathol Res Pract. 1998;194:797-803. doi:10.1016/S0344-0338(98)80070-8.

34. Maksymowych WP, Chiowchanwisawakit P, Clare T, Pedersen SJ, Ostergaard M, Lambert RG. Inflammatory lesions of the spine on magnetic resonance imaging predict the development of new syndesmophytes in ankylosing spondylitis: evidence of a relationship between inflammation and new bone formation. Arthritis rheumatism. 2009;60:93-102. doi:10.1002/art.24132.

35. van der Heijde D, Machado P, Braun J, Hermann KG, Baraliakos X. Hsu B, et al. MRI inflammation at the vertebral unit only marginally predicts new syndesmophyte formation: a multilevel analysis in patients with ankylosing spondylitis. Ann Rheum Dis. 2012;71:369-73. doi:10.1136/annrheumdis2011-200208.

36. Gorman JD, Sack KE, Davis JC. Treatment of ankylosing spondylitis by inhibition of tumor necrosis factor alpha. N Engl J Med. 2002;346:1349-56. doi:10.1056/NEJMoa012664.

37. Tseng HW, Pitt ME, Glant TT, McRae AF, Kenna TJ, Brown MA, et al. Inflammation-driven bone formation in a mouse model of ankylosing spondylitis: sequential not parallel processes. Arthritis research therapy. 2016;18:35. doi:10.1186/s13075-015-0805-0.

38. Brown MA, Kennedy LG, MacGregor AJ, Darke C, Duncan E, Shatford JL, et al. Susceptibility to ankylosing spondylitis in twins: the role of genes, HLA, and the environment. Arthritis rheumatism. 
1997;40:1823-8. doi:10.1002/art.1780401015.

39. Brown MA, Laval SH, Brophy S, Calin A. Recurrence risk modelling of the genetic susceptibility to ankylosing spondylitis. Ann Rheum Dis. 2000;59:883-6. doi:10.1136/ard.59.11.883.

40. Cortes A, Hadler J, Pointon JP, Robinson PC, Karaderi T, Leo P, et al. Identification of multiple risk variants for ankylosing spondylitis through high-density genotyping of immune-related loci. Nat Genet. 2013;45:730-8. doi:10.1038/ng.2667.

41. Australo-Anglo-American Spondyloarthritis C, Reveille JD, Sims AM, Danoy P, Evans DM, Leo P, et al. Genome-wide association study of ankylosing spondylitis identifies non-MHC susceptibility loci. Nat Genet. 2000;42:123-7. doi:10.1038/ng.513.

\section{Figures}

A

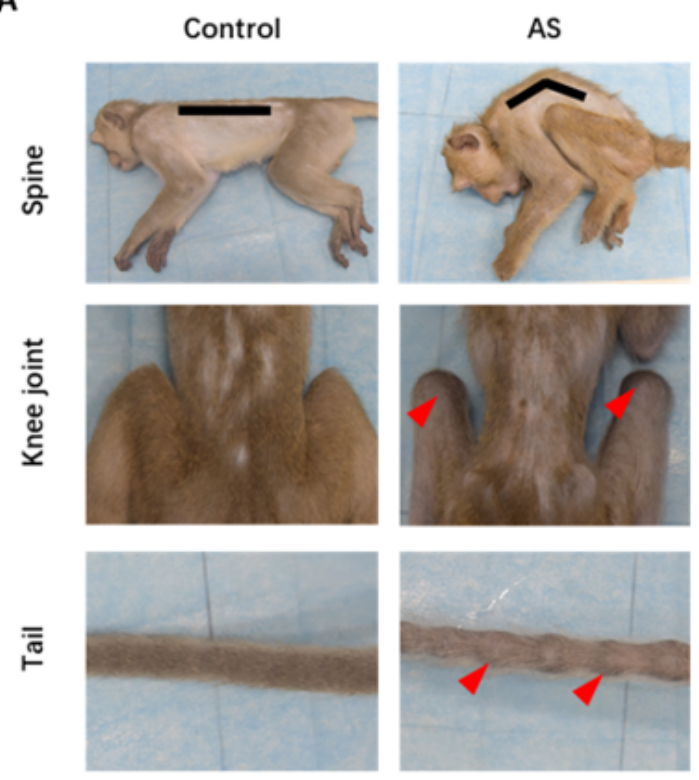

C

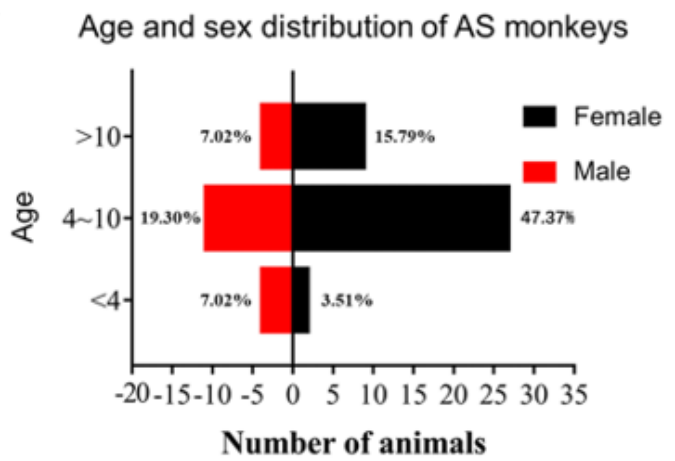

B
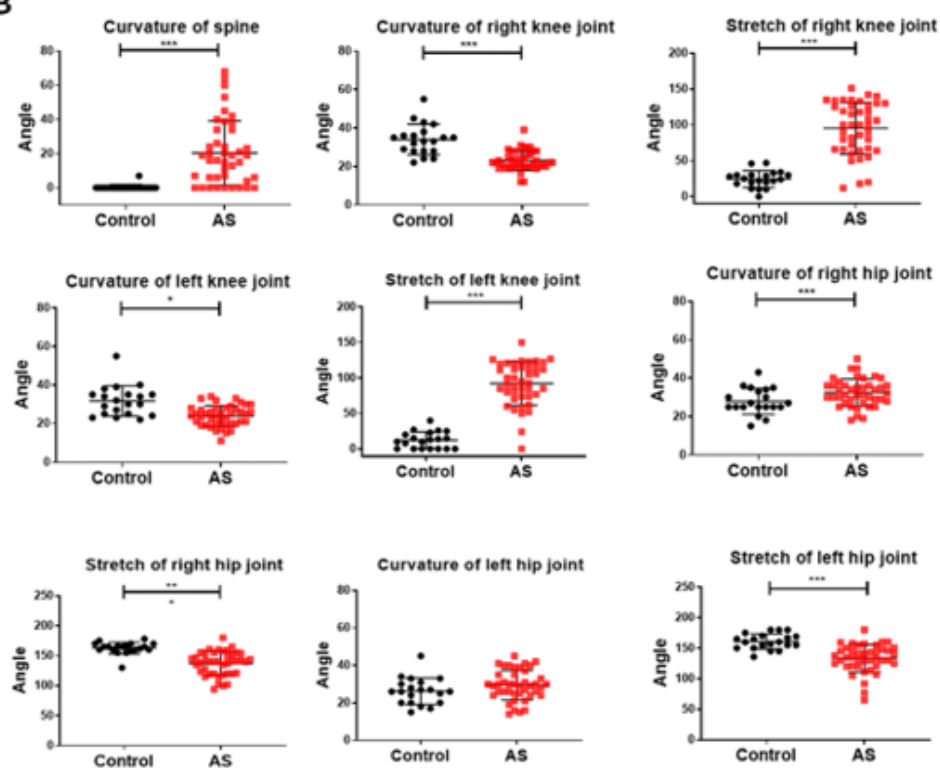

D

Family 1

Family 2
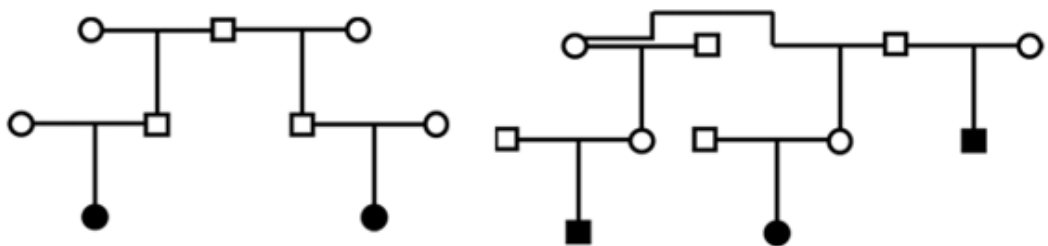

Male $\bigcirc$ Female $\square$ Male As

Female AS

\section{Figure 1}

Physical examination and epidemiology investigation of Cynomolgus monkeys. (A) Comparison of surface observations between normal and diseased animals. Representative images of cynomolgus 
monkeys spines, knees and tails. The spines of the diseased animal are curved (black line), the knee joints is swollen, the tail appears as nodular (red arrowhead). (B) Joints motilities were evaluated by a measuring ruler as descried in the methods. Values on graphs are presented mean \pm SD. $p$ values calculated by student's t-test, where ${ }^{\star} p<0.05,{ }^{\star \star \star} p<0.001$, compared with control (Control: $n=20$, AS: $n=42$ ). (C) Age and gender distribution of AS monkeys. The age of the animals counted in the figure is the age at which the animals were diagnosed $(n=57)$. (D) Family trees of 2 monkeys families. Squares $=$ males; circles = females; darkened squares and circles = monkeys with ankylosing spondylitis .

A
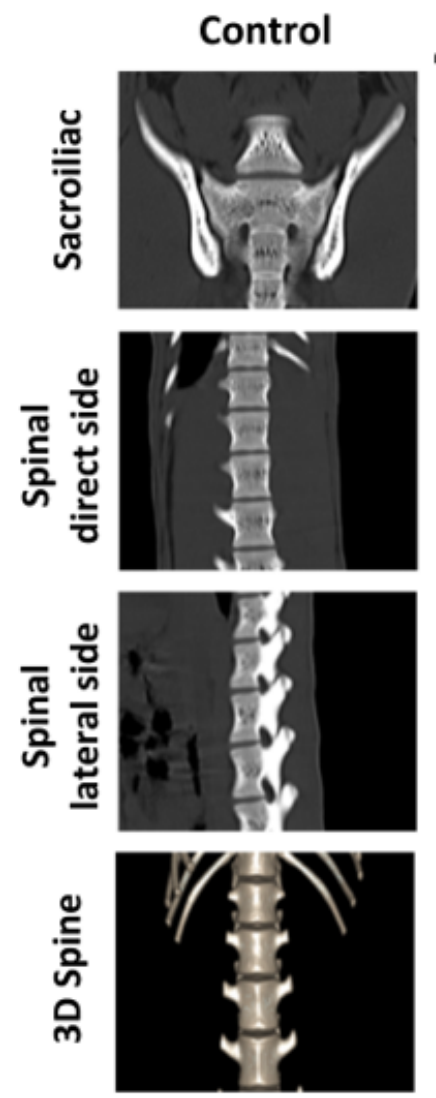

C

Control

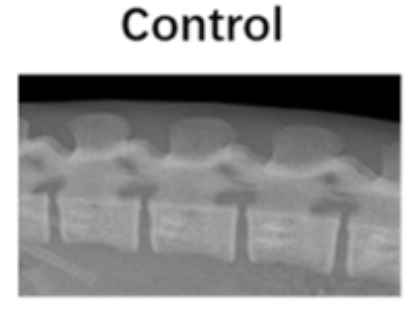

AS
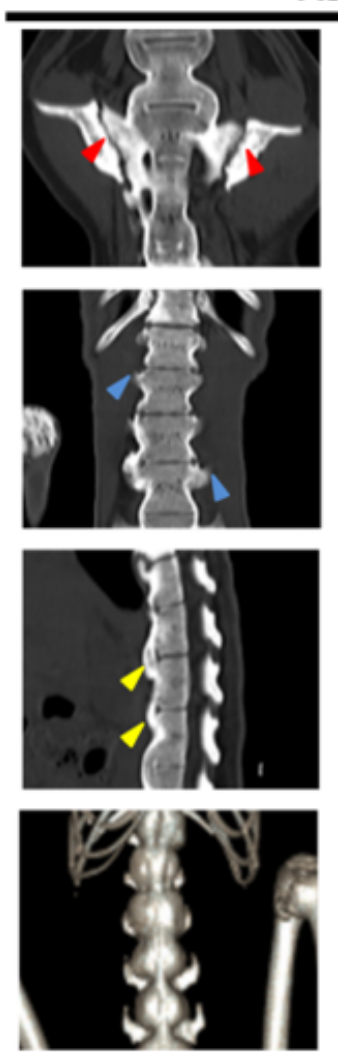

B
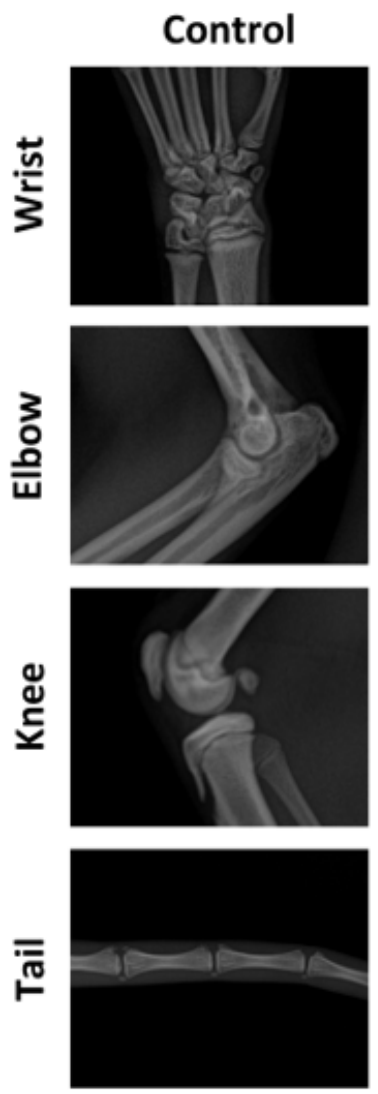

AS
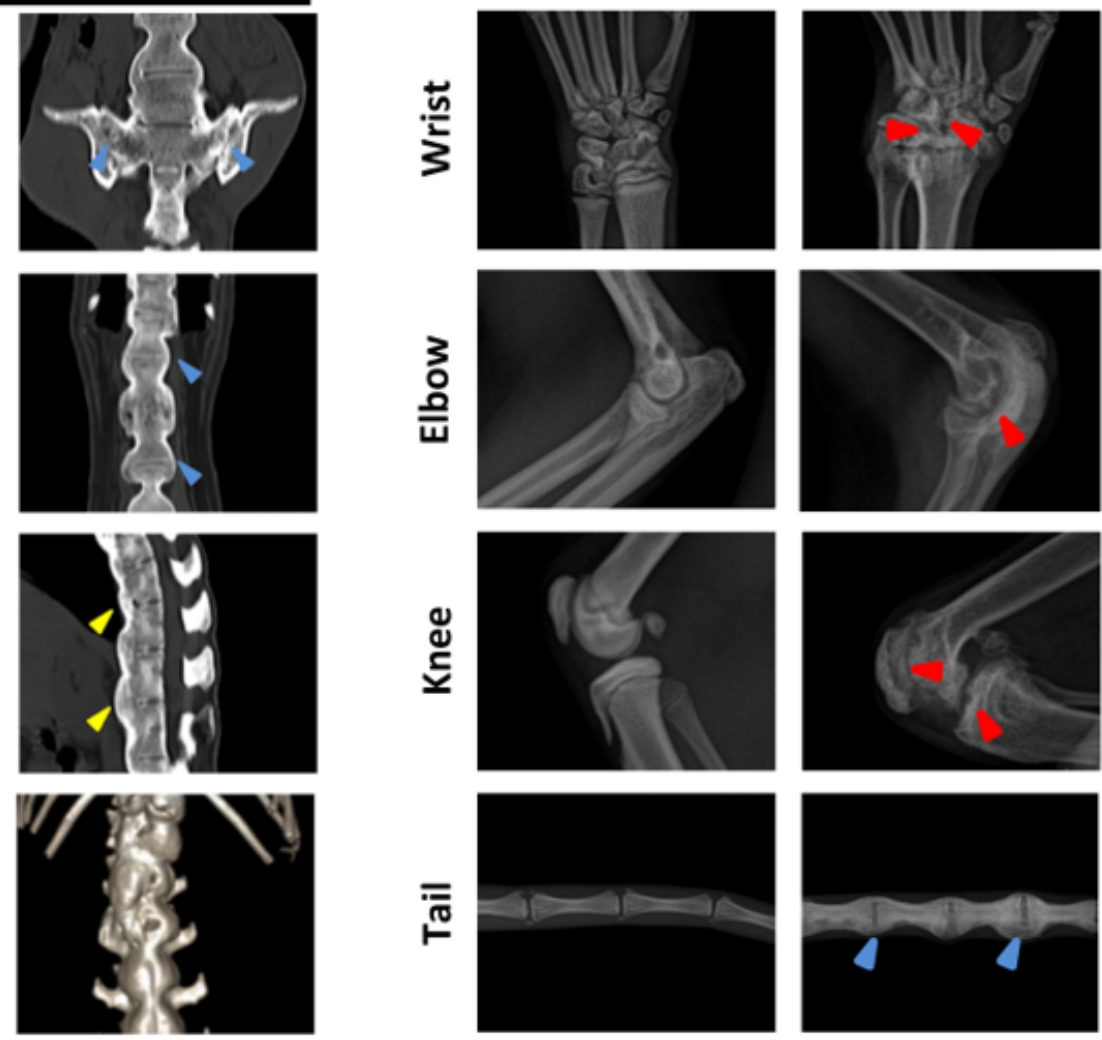
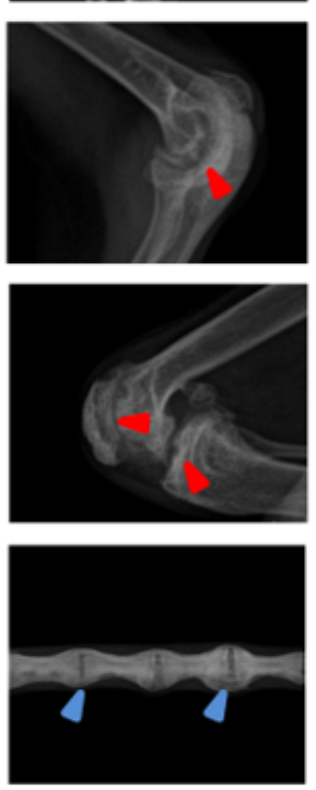

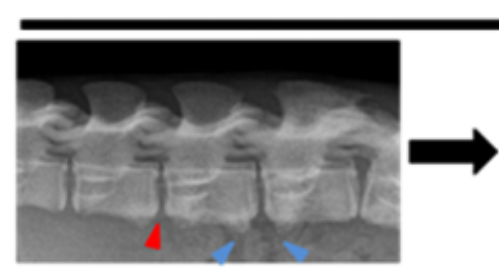

Erosion
AS

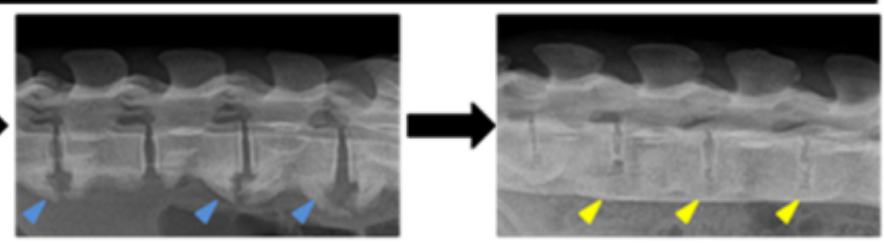

Bony bridge
Ligament

calcification

Figure 2

CT-scan and x-ray examinations of axial and peripheral joints in Cynomolgus monkeys. (A) Representative CT-scan images of sacroiliac joint and spine (L1-S1) of Cynomolgus monkeys. The AS 
monkeys showing extensive bone erosion (red arrowheads) and syndesmophte formation (blue arrowheads) in sacroiliac joint and spine. The bony spurs (yellow arrowheads) and vertebral bodies fuse together, the spine appears as a single piece and is described as bamboo spine (3D spine). (B) X-ray examinations of peripheral joints in Cynomolgus monkeys. Representative X-ray images of wrists, elbows, knees and tails of Cynomolgus monkeys, showing extensive bone erosion and/or syndesmophte formation (red arrowheads) in the AS monkeys. (C) Progression of radiographic lumbar joints of AS monkeys. The edges of vertebral bodies are blurred and slightly rough (red arrowheads) in early joint lesions. In the mid-term lesions, syndesmophte formation (blue arrowheads) is obviously as shown in the figure. In the late stage, the joint was fuse together (yellow arrowheads) and deformed.
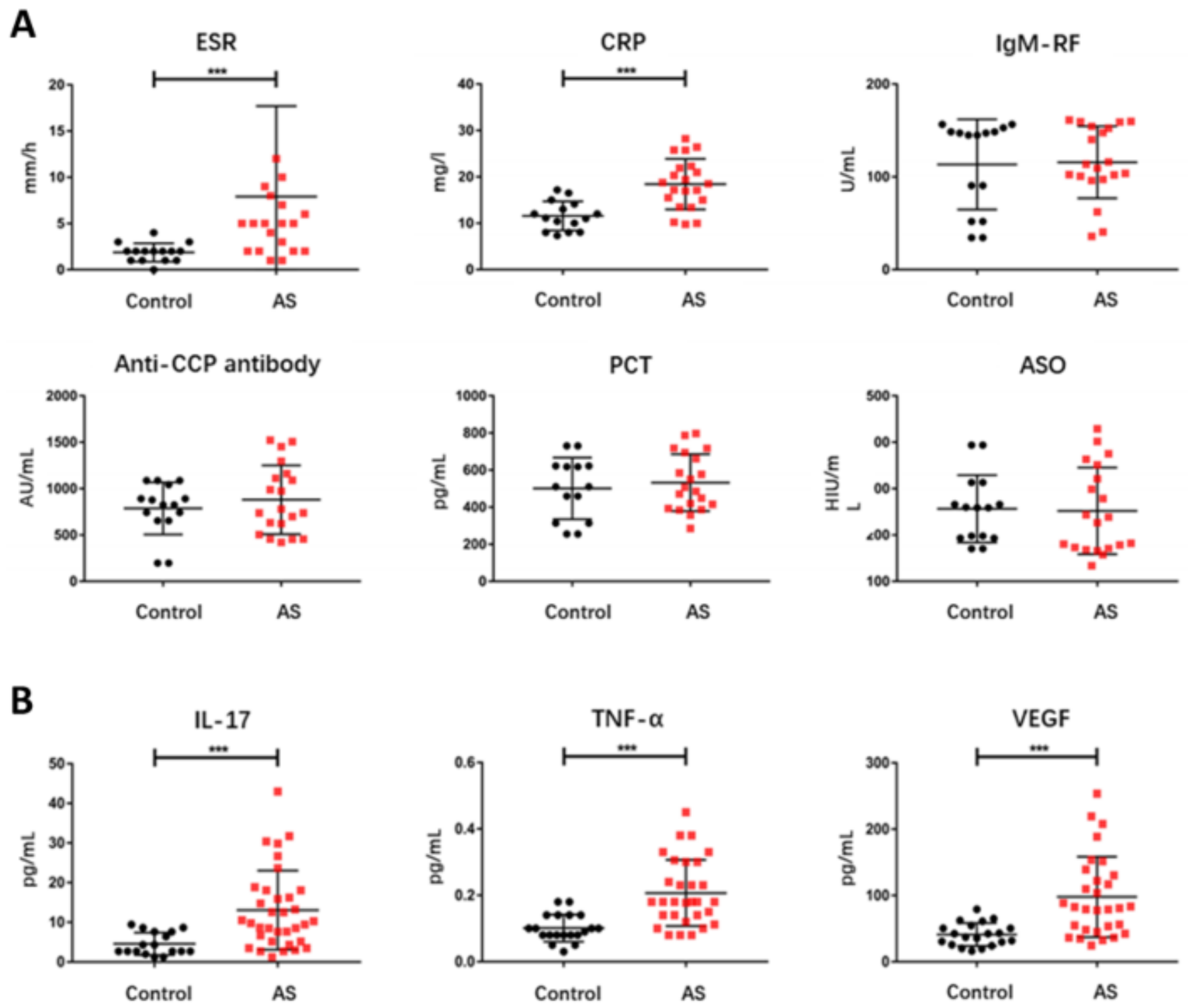

Figure 3

The hematological parameters and inflammatory cytokines of cynomolgus monkeys. (A) Evaluation of inflammatory cytokines of cynomolgus monkeys(Control: $n=15 \otimes A S: n=20$ ). The features of inflammatory 
cytokines include ESR (Erythrocyte sedimentation rate), CRP (C reactive protein), IgM-RF (Immunoglobulin M-rheumatoid factor), Anti-CCP antibody (Anti-cyclic peptide containing citrulline antibody ), PCT (Procalcitonin), ASO (Anti-Streptolysin 0). (B) Comparison of blood cell classification between normal and diseased cynomolgus monkeys (Control: $n=17, A S: n=34)$. The characteristic change of inflammatory cells is described by IL-17 (Interleukin 17), TNF-a (Tumor necrosis factor a) and VEGF (Vascular endothelial growth factor). Values on graphs are the mean \pm SD. $p$ values calculated by student's t-test, where $* P<0.05,{ }^{\star \star *} \mathrm{P}<0.001$, compared with control.
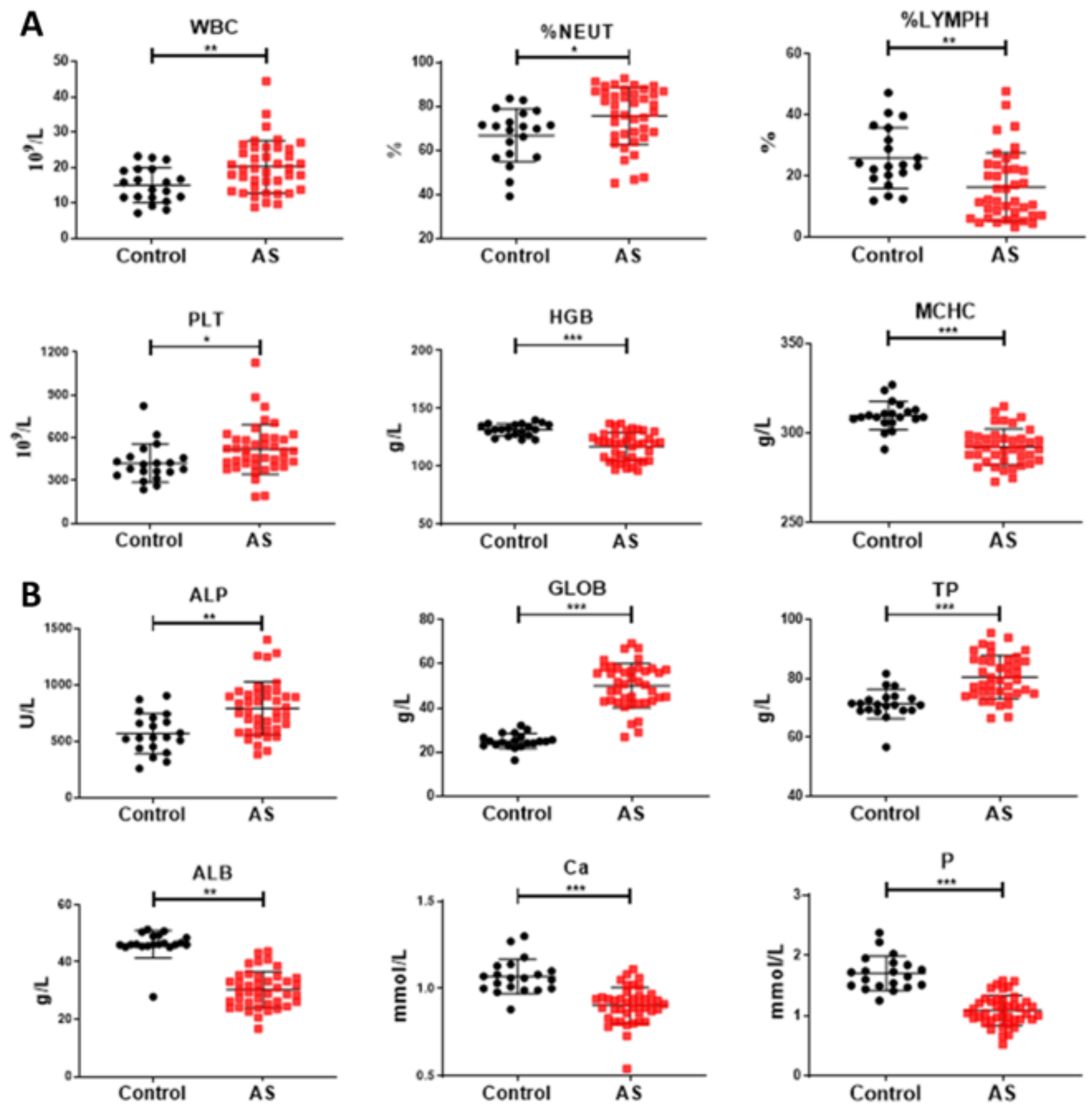

Figure 4 
Comparison of serum biochemistry between normal and diseased cynomolgus monkeys. (A) Inflammatory reaction is characterized as the change of WBC (White blood cell), \%NEUT (Percentage of neutrophils ), \%LYMPH (Percentage of lymphocyte), PLT(blood platelet), HGB (Hemoglobin) and MCHC (Mean corpuscular hemoglobin contentration) of diseased cynomolgus monkeys. (B) Serum ALP (Alkaline phosphatase), GLOB (Globulin), TP (Total Protein), ALB (Albumin), Ca (calcium) and P (phosphorus) of normal and diseased cynomolgus monkeys. Values on graphs are the mean \pm SD. $p$ values calculated by student's t-test, where $* P<0.05, * * P<0.01$, ***P $<0.001$, compared with control(Control: $n=20$, AS: $n=41$ )

A
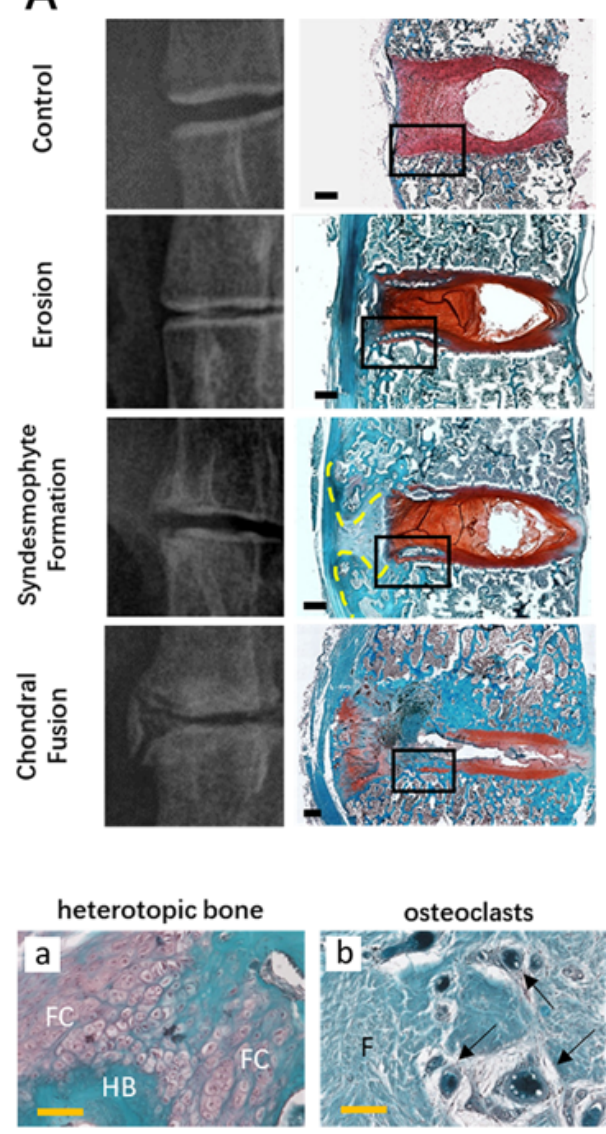

osteoclasts

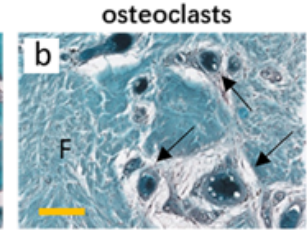

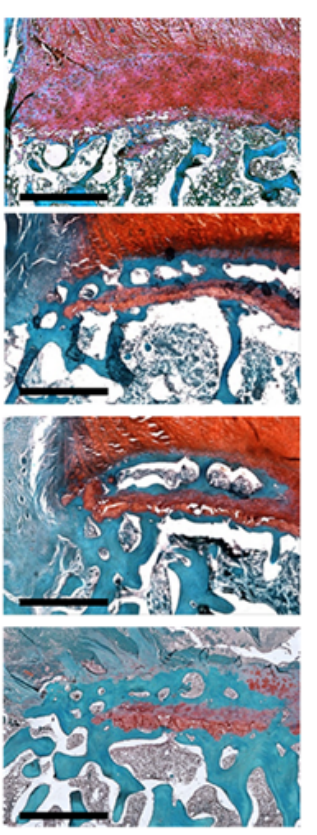

cartilage calcifications

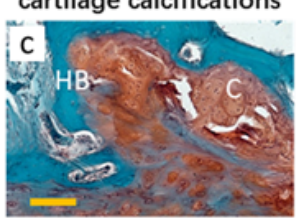

B
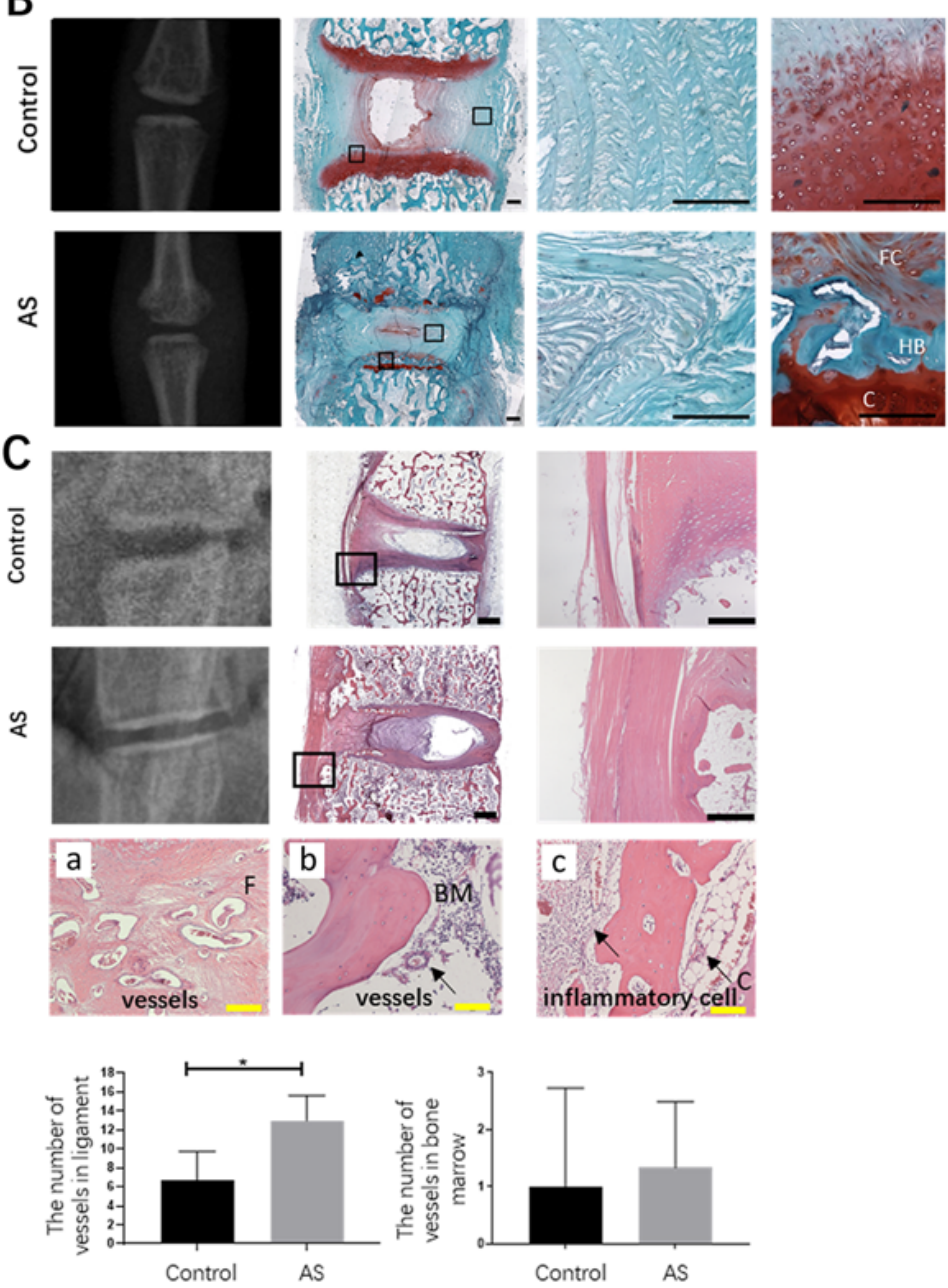

\section{Figure 5}

Histopathologic evaluation of ankylosed spine. (A) Histopathologic evaluation of ankylosed intervertebral joints. Spines from 6-10 years old normal and diseased animals $(n=3)$ were harvested, fixed, and stained. The bone is stained blue while the cartilage and annulus fibrosus are stained red. Representative safranin-0/fast green images of normal animals joint demonstrating normal joint cavity, healthy cartilage, and annulus fibrosus. Representative safranin- $\mathrm{O} /$ fast green of diseased animals intervertebral joints demonstrating cartilage disruption of the disc by a heterotopic ossification tissue (Magnification of the boxed area in Erosion vertebrae shows bony invasion and cartilage damage.) and heterotopic bone formation was shown at the edge of the vertebral bodies. The yellow dotted line indicates the 
Syndesmophyte surface. Representative safranin-0/fast green of diseased animals syndesmophyte demonstrating heterotopic bone, fibrocartilage, osteoclasts(arrows) and cartilage calcifications(c). FC fibrocartilage, $\mathrm{F}$ fibrous tissue, $\mathrm{HB}$ heterotopic Bone, $\mathrm{C}$ cartilage plate. Black scale bar $=1 \mathrm{~mm}$. Yellow scale bar $=100 \mu \mathrm{m}$. (B) Representative safranin-O/fast green image of normal tail joint demonstrating normal joint cavity, healthy cartilage, and annulus fibrosus. Magnification of the boxed area showed irregularly organized annulus fibrosus, fibrocartilage and heterotopic bone in the surface of cartilage from monkeys with AS. FC fibrocartilage, HB heterotopic bone, C cartilage. Scale bar $=200 \mu \mathrm{m}$. (C) Representative HE images and the number of vessels of normal animals and diseased animals joints. Magnification of the boxed area showed the incrassation of ligament, inflammatory cell infiltration(c), and the vessels in fibrosus tissue(a) and bone marrow(b) from monkeys with AS. F fibrous tissue, BM bone marrow, $\mathrm{C}$ cartilage plate, $\mathrm{A}$ annulus fibrosus. Black scale bar $=500 \mu \mathrm{m}$. Yellow scale bar $=100 \mu \mathrm{m}$. The number of vessels in ligament from monkeys with AS is significantly higher than normal animals. (Control: $n=3, A S: n=3$ )
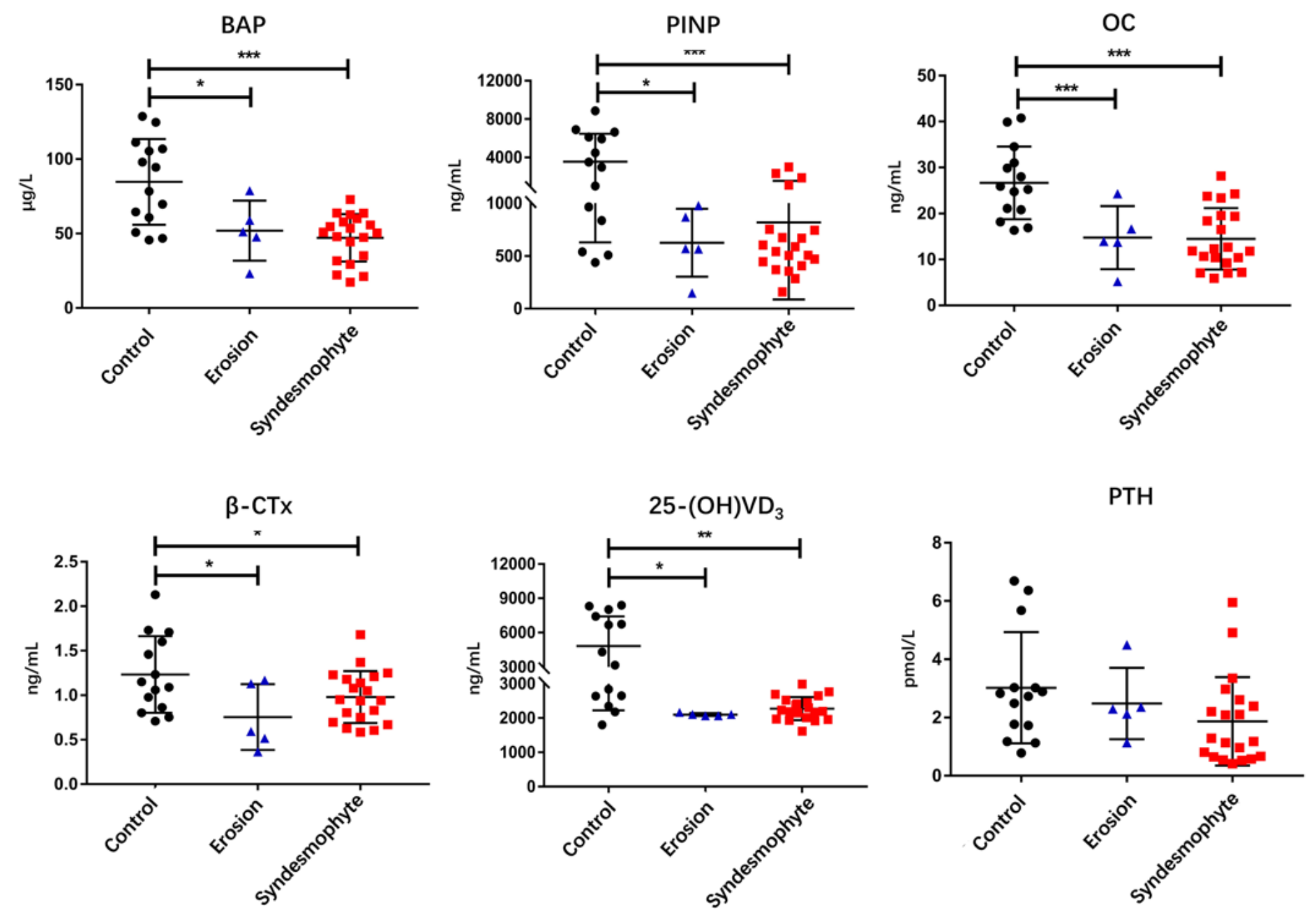

Figure 6

Indexs of bone metabolism of cynomolgus monkeys. Erosion represent the subgroup of AS monkeys with erosion in the margin of vertebrae. Syndesmophyte represent the subgroup of AS monkeys with 
syndesmophyte formation in vertebrae. These two subgroups were divided according to their X-ray results. Bone formation indicators: Bone-specific alkaline phosphatase (BAP), N-terminal propeptide of type I Procollagen (PINP) and Osteocalcin (OC). Bone resorption indicators: $\beta$-C-terminal telopeptides of type I collagen ( $\beta$-CTx), 25-(OH)VD3(25-hydroxyvitamin D3) and PTH (parathyroid hormone). Values on graphs are the mean $\pm S D$. $P$ values calculated by student's t-test, where $* P<0.05$, ** $P<0.01$, *** $P<$ 0.001 , compared with control. (Control: $n=14$, Erosion: $n=5$, Syndesmophyte: $n=20$ ) 